\title{
Beweis eines Satzes über zwei cubische Raumcurven, welche dasselbe Tetraeder in gleicher Weise zum Schmiegungstetraeder haben.
}

\author{
Von Gustav Kohn in Wien.
}

Cubische Raumcurven, welche zwei Punkte $A_{1}$ und $A_{2}$ und zugleich deren Tangenten und Schmiegungsebenen gemein haben, bilden ein Analogon zu Kegelschnitten in doppelter Berührung. $\mathrm{Zu}$ dieser schon mehrfach hervorgehobenen Analogie liefert der folgende Satz einen Beitrag.

Die Verbindungsebenen der Tripel entsprechender Punkte von drei projectiven Punktreihen auf einer cubischen Raumcurve $R_{3}$, in welchen die zwei Punkte $A_{1}$ und $A_{2}$ sich selbst entsprechen, umhüllen eine zweite cubische Raumcurve $R_{3}^{\prime}$, welche mit der ersten die Punkte $A_{1}$ und $A_{2}$ und in ihnen Tangenten und Schmiegungsebenen gemein hat; umgekehrt schneiden die Schmiegungsebenen jeder-solchen cubischen Raumcurve auf $R_{3}$ Tripel entsprechender Punkte von drei projectiven Reihen aus, in denen die Punkte $A_{1}$ und $A_{2}$ sich selbst zugeordnet erscheinen."

In den folgenden Zeilen soll ein synthetischer Beweis dieses Satzes erbracht werden und zwar auf Grund der Theorie der Correlationen.

Für eine beliebige Correlation des Raumes erfüllen bekanntlich die Punkte, welche auf ihren entsprechenden Ebenen liegen, eine Fläche zweiter Ordnung $F_{2}$ (die Punktkernfläche) und diese Ebenen, d. h. die Ebenen, welche durch ihre entsprechenden Punkte gehen, umhüllen eine zweite solche Fläche $\Phi_{2}$ (die Ebenenkernfläche). Die beiden Flächen $F_{2}$ und $\Phi_{2}$ durchdringen sich in den Kanten eines windschiefen Vierkants, der Hauptvierkants der Correlation, dessen Kanten sich als die Geraden charakterisieren lassen, welche sich in der Correlation selbst entsprechen. Die Annahme des Hauptvierkants für eine Correlation ist äquivalent mit der Annahme, dass jedem der vier Eckpunkte des Vierkants die Verbindungsebene der in ihm zusammenstofenden Kanten als entsprechend zugeordnet ist. Die Correlation ist also durch Annahme 
ihres Hauptrierkants noch nicht bestimmt und es kann zu ihrer Bestimmung noch einem beliebigen Punkte des Raumes die entsprechende Ebene willkürlich zugewiesen werden.

Es sei jetzt ein beliebiges windschicfes Vierkant vorgelegt und $t_{1}, t_{2}$ seien zwei seiner Gegenkanten, $s_{1}, s_{2}$ die beiden anderen. Der Schnittpunkt der Kanten $s_{1}$ und $t_{1}$ heiße $A_{1}$, ihre Verbindungsebene $\alpha_{1}$, der Schnittpunkt von $s_{2}$ und $t_{2}$ sei mit $A_{2}$, die Verbindungsebene mit $\alpha_{2}$ bezeichnet. Die cubischen Raumcurren, welche durch die beiden Punkte $A_{1}$ und $A_{2}$ hindurchgehen und in ihnen beziehungsweise die Geraden $t_{1}$ und $t_{2}$ zu Tangenten, die Ebenen $\alpha_{1}$ und $\alpha_{2}$ zu Schmiegungsebenen haben, bilden ein Bündel von cubischen Raumcurven, welche dasselbe Tetraeder, nämlich das Tetraeder des Vierkants, in derselben Weise zum Schmiegungstetraeder haben. Von den Curven dieses Bündels geht bekanntlich durch einen beliebigen Punkt des Raumes eine und eine beliebige Ebene ist Schmiegungsebene für eine unter diesen Curven. Man erkennt sofort, dass jede Correlation des Raumes, welche das Vierkant $t_{1} s_{1} t_{2} s_{2}$ zum Hauptvierkant hat, die Curven unseres Bündels lediglich unter einander vertauscht.

Um alle Vorbedingungen für unseren Beweis vor Augen zu haben, beachten wir endlich noch, dass die Fläche zweiter Ordnung, welche eine beliebige Curve $R_{3}$ unseres Bündels mit ihren Tangenten $t_{1}$ und $t_{2}$ verbindet, auch die Strahlen $s_{1}$ und $s_{2}$ enthalten wird. In der That enthält diese Fläche jede Transversale, welche durch einen beliebigen Punkt $P$ der Curve $R_{3}$ hindurchgeht and die beiden Geraden $t_{1}, t_{2}$ trifft und diese Transversale geht in die Gerade $s_{1}$ resp. $s_{2}$ über, sobald der Punkt $P$ in den Punkt $A_{1}$ resp. $A_{2}$ hineinrückt. Denn die Gerade $s_{1}$ (bez. $s_{2}$ ) liegt unseren Voraussetzungen gemäß, in der Verbindungsebene des zu $P_{1}$ (bez. $P_{2}$ ) unendlich nahen Curvenpunktes mit seiner Tangente $t_{1}$ (bez. $t_{2}$ ) d. h. in der Schmiegungsebene von $P_{1}$ (bez. $P_{2}$ ) und trifft auferdem die Gerade $t_{9}$ (bez, $t_{1}$ ). Wir können jetzt sagen, dass die Fläche zweiter Ordnung, welche den Kantenzug $t_{1} s_{1} t_{2} s_{2}$ mit einem beliebigen Punkte der Curve $R_{3}$ verbindet, die ganze Curve enthält.

Wir bestimmen nun eine correlative Beziehung zweier Räume, für welche das Vierkant $t_{1} s_{1} t_{2} s_{2}$ das Hauptvierkant darstellt, indem wir im ersten Raume einen Punkt $P$ beliebig aunehmen und ihm eine durch ihn hindurchgehende, sonst beliebige Ebene $\pi^{\prime}$ als entsprechend zuweisen. Der Punkt $P$ wird dann auf der Punktkernfläche der Correlation liegen, welche deshalb bestimmt erscheint, als die Fläche zweiter Ordnung $F_{2}$, die diesen Punkt mit dem Kantenzuge $t_{1} s_{1} t_{2} s_{2}$ verbindet. Die cubische Raumeurve $R_{3}$ unseres Bündels, welche durch den Punkt $P$ geht, wird ganz auf dieser Fläche $F_{2}$ liegen und deswegen wird jedem Punkte dieser Curve in der Correlation eine Ebene entsprechen, welche durch ihn hindurchgeht. Die den Punkten der Curve $R_{3}$ unseres Bündels entsprechenden. Ebenen werden eine zweite cubische Raum- 
curve $R_{3}^{\prime}$ dieses Bündels einhüllen, nämlich jene, welche die dem Punkte $P$ entsprechende Ebene $\pi^{\prime}$ zur Schmiegungsebene hat.

Nun lassen sich $R_{3}$ and $R_{3}^{\prime}$ als zwei ganz beliebige Curven unseres Bündels ansehen. Denn man kann den Punkt $P$ unserer Betrachtung beliebig auf der Curve $R_{3}$ wählen und hat ihm dann irgend eine der drei Schmiegungsebenen als entsprechende Ebene $\pi^{\prime}$ zuzuweisen, die er an die Curve $R_{3}^{\prime}$ schickt. Es gibt infolge dessen drei Correlationen, welche die Punkte ron $R_{3}$ auf die Schmiegungsebenen von $R_{3}^{\prime}$ so abbilden, dass jeder Punkt in der ihm entsprechenden Ebene liegt. Die Reihe der Punkte der Curve $R_{3}$ erscheint so in dreifacher Weise auf die Reihe der Schmiegungsehenen von $R_{3}^{\prime}$ bezogen, dass in diesen drei projectiven Beziehungen einem beliebigen Punkte von $R_{3}$ die drei durch ihn gehenden Schmiegungsebenen von $R_{3}^{\prime}$ einer beliebigen Schmiegungsebene von $R_{3}^{\prime}$ die drei in ihr liegenden Punkte von $R_{3}$ entsprechen, und für jeden der beiden Punkte $A_{1}$ und $A_{2}$, in welchen die beiden Curven $R_{2}$ und $R_{3}^{\prime}$ gemeinsame Schmiegungsebenen haben, wird offenbar die entsprechende Ebene mit der zugehörigen Schmiegungsebene zusammenfallen.

Jetzt ist also einerseits deutlich, dass die drei Schnittpunkte einer veränderlichen Schmiegungsebene von $R_{3}^{\prime}$ mit der Curve $R_{3}$ auf dieser Curve drei zur Reihe der Schmiegungsebenen von $R_{3}^{\prime}$ also auch zu einander projectiver Punktreihen durchlaufen und dass in diesen drei projectiven Punktreihen auf der Curve $R_{3}$ jeder der beiden Punkte $A_{1}$ and $A_{2}$ sich selbst entspricht. Es ist aber auch anderseits $\mathrm{zu}$ sehen, dass für irgend welche drei projective Punktreihen auf der Curve $R_{3}$, in denen jeder der beiden Punkte $A_{1}, A_{2}$ sich selbst entspricht, die Verbindungsebenen der Tripel entsprechender Punkte eine cubische Raumeurve umhüllen werden, welche mit $R_{3}$ die Punkte $A_{1}$ und $A_{2}$ und die ihnen Schmiegungsebenen und 'Tangenten gemein hat. Denn, wenn neben den beiden sich selbst entsprechenden Punkten $A_{1}$ und $A_{3}$ noch ein Tripel entsprechender Punkte gegeben wird, so sind die drei projectiven Punktreihen auf $R_{3}$ bestimmt. Die Ebene dieser drei Punkte wird nun von einer cubischen Raumeurve $R_{3}^{\prime}$ osculiert, welche die Punkte $A_{1}$ und $A_{2}$ nebst ihren Schmiegungsebenen und Tangenten mit $R_{3}$ gemein hat und die Schmiegungsebenen von $R_{3}^{\prime}$ schneiden auf $R_{3}$ die Tripel entsprechender Punkte aus. 